\title{
A Truncated, Secreted Form of the Epidermal Growth Factor Receptor Is Encoded by an Alternatively Spliced Transcript in Normal Rat Tissue
}

\author{
LESLIE A. PETCH,${ }^{1}$ JOANNA HARRIS, ${ }^{2}$ VICTORIA W. RAYMOND ${ }^{3}$ ANDREW BLASBAND,${ }^{2}$ DAVID C. LEE, ${ }^{2,4}$ \\ AND H. SHELTON EARP ${ }^{1,2,5 *}$ \\ Departments of Pharmacology, ${ }^{1}$ Pathology, ${ }^{3}$ Medicine, ${ }^{5}$ Microbiology and Immunology, ${ }^{4}$ and Cancer Cell Biology \\ Program, ${ }^{2}$ Lineberger Cancer Research Center, University of North Carolina at Chapel Hill, Chapel Hill, \\ North Carolina 27599
}

Received 7 November 1989/Accepted 20 March 1990

\begin{abstract}
Two independent cDNA clones corresponding to a 2.7-kilobase (kb) epidermal growth factor receptor (EGF-R) mRNA were isolated from a rat liver cDNA library. Sequence analysis revealed $100 \%$ homology in the external domain when compared with the full-length rat EGF-R nucleotide sequence and 80 to $90 \%$ similarity relative to the human EGF-R. However, the 3 '-terminal sequence of these clones did not match EGF-R or any other known sequence(s) and was distinct from the $3^{\prime}$ end of the 2.8-kb mRNA, which encodes a truncated EGF-R in A431 cells. The deduced amino acid sequence revealed an open reading frame which is homologous to the external domain of the EGF-R but which terminates prior to the transmembrane region. Southern blot analysis of rat genomic DNA indicated that the $3^{\prime}$-terminal sequence of this transcript is derived from the EGF-R gene. Analysis of a genomic clone containing the $3^{\prime}$ end of the 2.7-kb transcript revealed that this sequence is present as a discrete exon in the mid-region of the receptor gene in proximity to the exon encoding the transmembrane domain. Introduction of an expression vector containing the truncated EGF-R cDNA into Chinese hamster ovary (CHO) cells led to the expression of a 95-kilodalton protein which was detected in conditioned media, by using antisera directed against the EGF-R. A similarly sized protein was also detected in the media of WB cells, a continuous, nontransformed line of rat hepatic epithelial cells. Northern (RNA blot) analysis established that the truncated receptor is encoded by a 2.7-kb transcript found in normal rat liver. Furthermore, Northern analysis of rat poly $(A){ }^{+}$RNA showed that the $2.7-k b$ EGF-R transcript is expressed at differing levels in various fetal and adult tissues. These data indicate that alternative splicing of the EGF-R primary transcript yields a $2.7-\mathrm{kb}$ mRNA which codes for a truncated form of the receptor. This receptor is secreted by rat hepatic epithelial cells in culture, which suggests that it may be secreted by normal rat cells or tissues and perhaps serve an as yet unknown physiological function.
\end{abstract}

Control of cell growth and proliferation involves a complex series of events initiated by the interaction of serum growth factors with their cell surface receptors. A large number of these cell surface receptors belong to a family of growth factor receptors which consist of an extracellular ligand-binding domain linked to a cytoplasmic domain that possesses intrinsic tyrosine kinase activity (for current reviews, see references 3 and 50). One member of this family, the epidermal growth factor receptor (EGF-R), is a 170kilodalton $(\mathrm{kDa})$ glycoprotein that mediates the effects of both EGF and transforming growth factor alpha. Stimulation of the EGF-R by these factors leads to a variety of early and delayed biochemical and physiological responses which result in the proliferation of target cells (for a review, see references 3, 4, and 50). Early responses to growth factor stimulation include protein phosphorylation at tyrosine residues, activation of transport systems, and, in some cell lines, stimulation of phosphoinositide turnover. This is followed several hours later by an increase in specific protein and RNA synthesis, and by 12 to $18 \mathrm{~h}$, DNA synthesis ensues. Binding of ligand to the receptor leads to receptor clustering on the cell surface followed by the internalization of the growth factor-receptor complex and its subsequent degradation in lysosomes. The biological role of receptor internalization and the mechanism through which EGF and

\footnotetext{
* Corresponding author.
}

transforming growth factor alpha stimulate DNA synthesis and cell proliferation are not yet understood.

The EGF-R gene is the cellular homolog of the v-erbB oncogene (7). This proto-oncogene can be activated by insertional mutagenesis following avian leukosis virus infection $(11,23)$ or as a result of transduction by the avian erythroblastosis virus $(13,16,22,32,49)$. In both cases, the product is a truncated receptor which lacks a ligand-binding domain and, in the case of avian erythroblastosis virus, it also lacks the C-terminal autophosphorylation sites (for a review, see reference 28 ). The absence of these regulatory domains could potentially lead to constitutive activation of the tyrosine kinase and ultimately result in transformation. Altered forms of the EGF-R gene have also been observed in a variety of human primary tumors and tumor cell lines of glial and squamous origin $(10,15,17,18,26,34,48)$. These alterations, which usually involve amplification of the gene and which may or may not be accompanied by gene rearrangement, result in overexpression of the EGF-R protein. Both amplification and rearrangement of the EGF-R gene have been observed in A431 epidermoid carcinoma cells (27, $31,38,43,47)$. These cells produce an unusually high number of EGF-Rs in addition to secreting a truncated form of the protein $(9,45)$. This truncated receptor correlates with the expression of a 2.8-kilobase (kb) mRNA which diverges from the major EGF-R transcripts found in other human cell types $(10.5$ and $5.8 \mathrm{~kb})$ at its extreme $3^{\prime}$ end $(30,43)$. 
A.

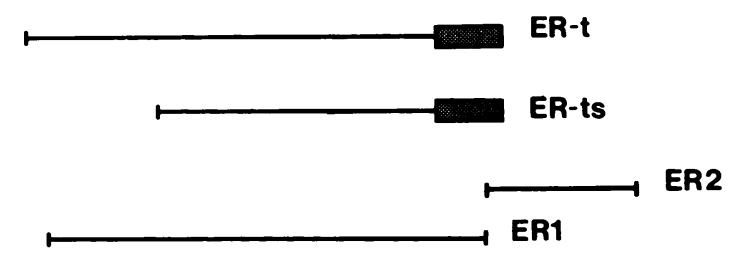

B.
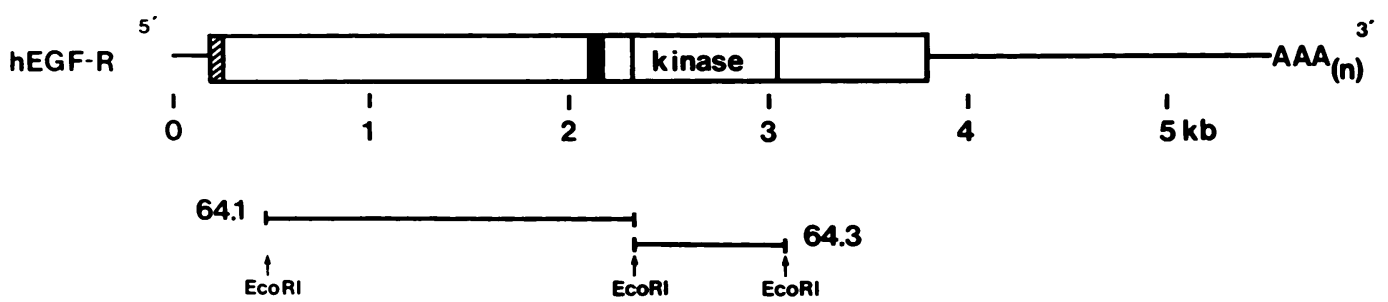

FIG. 1. Schematic diagram of the human and rat EGF-R cDNA clones. (A) Schematic representation of the rat EGF-R cDNA clones as compared with the human EGF-R cDNA. The inserts from plasmids pER-t and pER-ts represent duplicate clones of the truncated rat EGF-R. Clones ER1 and ER2 represent contiguous cDNAs derived from the full-length receptor mRNA. Horizontal lines represent the regions of homology to the human EGF-R. Stippled boxes represent the divergent sequence at the $3^{\prime}$ end of the truncated receptor cDNAs. (B) Schematic map of the full-length human EGF-R (hEGF-R) cDNA (43). Untranslated sequences are represented by a line; coding sequences are represented by an open bar. The hatched box at the $5^{\prime}$ end of the coding region represents the signal peptide; the black box represents the transmembrane domain. (n) indicates an unknown number of A residues in the poly(A) tract. The human cDNA clones (64.1 and 64.3) used as hybridization probes to screen the rat liver cDNA library are represented by horizontal lines.

Analysis of the EGF-R gene in A431 cells had suggested that the truncated mRNA is created by a gene rearrangement resulting in the fusion of the $5^{\prime}$ portion of the receptor gene to an unidentified region of genomic DNA (30).

Here, we report the cloning of a similar transcript from a rat liver cDNA library and provide evidence suggesting that alternative splicing of the full-length EGF-R transcript in normal cells generates a truncated form of the receptor. This truncated form lacks a transmembrane region and is, therefore, secreted. We also show that this transcript is expressed in a variety of normal tissues and that a truncated form of the EGF-R is expressed and secreted from WB cells, a continuous, nontransformed line of rat hepatic epithelial cells. We conclude that this truncated receptor from rat liver is neither the result of a gene rearrangement nor a product of the transformed phenotype but may, in fact, have a physiological function.

\section{MATERIALS AND METHODS}

cDNA and genomic cloning. A cDNA library was prepared as described previously (24) from mRNAs derived from rat liver by using a $\lambda$ gt10 vector system. The library was screened with $5^{\prime}$ - or $3^{\prime}$-encoding human EGF-R cDNAs (clones 64-1 and 64-3, respectively; kindly provided by $A$. Ullrich; see Fig. 1 for schematic representation). Four positive clones were obtained $(0.7,1.6,2.2$, and $2.3 \mathrm{~kb}$ in size) and subcloned into pEMBL (plasmids pER2, pER-ts, $\mathrm{pER} 1$, and $\mathrm{pER}-\mathrm{t}$, respectively). Plasmid DNA was purified by $\mathrm{CsCl}$ centrifugation, and the cDNA inserts were sequenced by the dideoxynucleotide chain termination method (35). Plasmid pMB122.6ER was made by ligating the $2.3-\mathrm{kb}$ rat EGF-R insert from pER-t into the EcoRI site of the retroviral expression vector pMB122.6 (kindly provided by R. Snodgrass), which also contains the bacterial neomycin resistance gene.

A rat genomic library produced in bacteriophage Charon
4A (CLONTECH) was screened in duplicate with the $3^{\prime} 1.2$ $\mathrm{kb}$ of cDNA clone ER-ts and $\mathrm{a}^{32} \mathrm{P}$-labeled oligonucleotide, specific for the 3'-terminal end of ER-t and ER-ts (Fig. 2; nucleotides 2259 to 2308). Bacteriophage DNA was prepared and sequenced as previously described (41).

Cells and transfections. CHO cells were grown in Eagle minimal essential medium with $10 \%$ bovine calf serum. Transfections were by the calcium phosphate precipitation method as previously described $(14,46)$. The cells were subsequently selected with genetecin G418 $(0.5 \mu \mathrm{g} / \mathrm{ml}$; GIBCO). Resistant colonies were picked after 2 weeks and screened for EGF-R mRNA expression by Northern (RNA) blot analysis. Positive clones were tested for expression of truncated EGF-R by immunoprecipitation of $\left[{ }^{35}\right.$ S]cysteineor $\left[{ }^{35} S\right]$ methionine-labeled receptors from conditioned media or tunicamycin-treated cells, with an antiserum (Ab 1382) to the rat EGF-R.

RNA and DNA blot analysis. Total cellular RNA was isolated from confluent $\mathrm{CHO}$ cells grown on $100-\mathrm{mm}$ plates by the method of Glisin et al. (12). Cells were lysed in guanidinium isothiocyanate, and the resulting lysates were centrifuged over a $\mathrm{CsCl}$ cushion. RNA from rat liver, uterus, decidua, and embryo was isolated by the method of Chirgwin et al. (5), and poly $(A)^{+}$was selected by chromatography on oligo(dt) cellulose. Total RNA $(40 \mu \mathrm{g})$ or poly $(A)^{+}$selected RNA ( $5 \mu \mathrm{g}$ per lane) was subjected to $1.0 \%$ agarose gel electrophoresis in formamide and was transferred to nitrocellulose (42). The RNA blots were probed with human EGF-R clones 64.1 and 64.3, nick-translated cDNA inserts from pER-ts, pER1, and pER2, or an end-labeled 50-basepair (bp) synthetic oligonucleotide specific to the $3^{\prime}$ end of EGF-R clone ER-t.

DNA isolated from Sprague-Dawley rat liver cells (10 to $20 \mu \mathrm{g}$ ) was digested with the appropriate restriction enzymes, subjected to $0.7 \%$ agarose gel electrophoresis, and transferred to nitrocellulose by the method of Southern (40). 


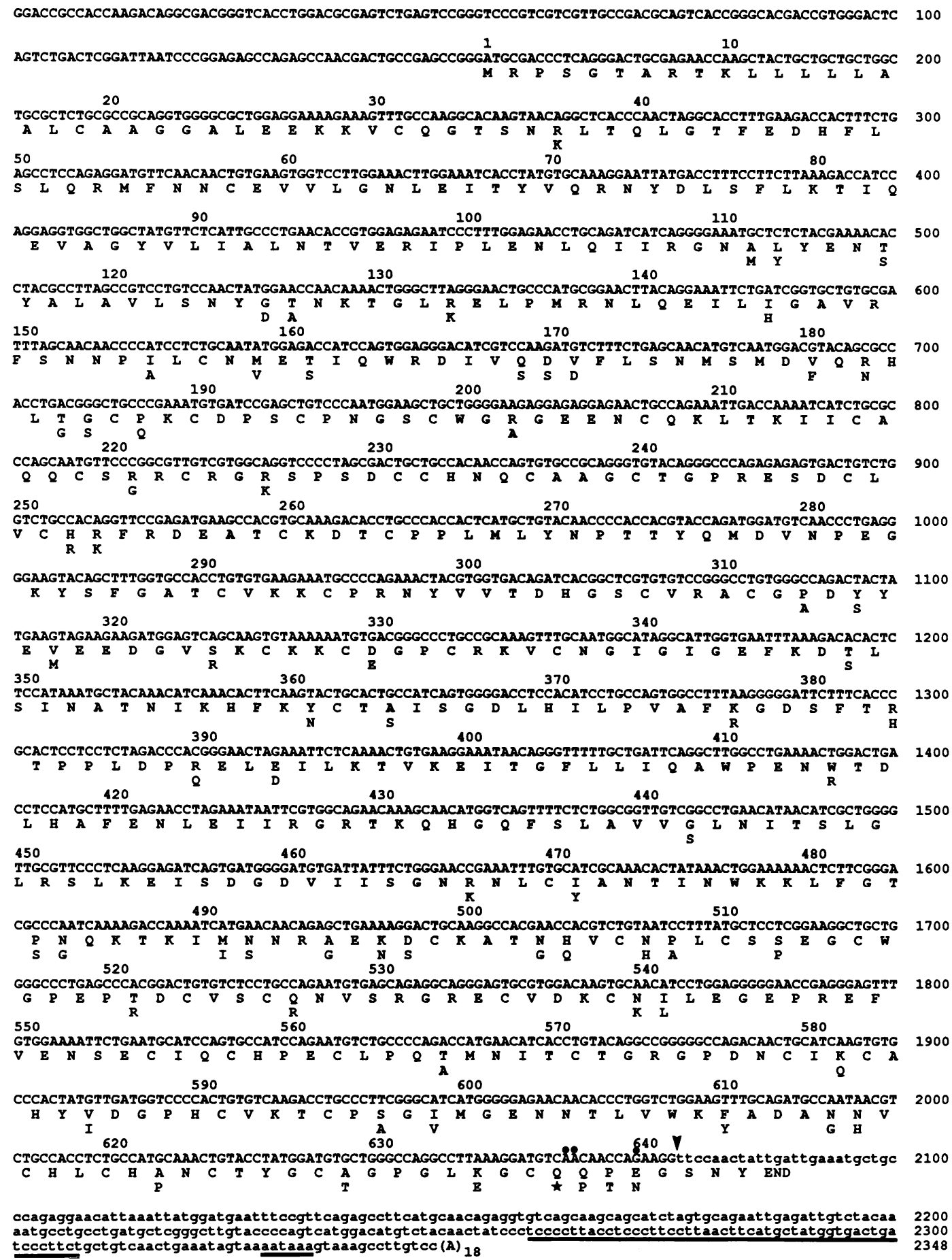

FIG. 2. Nucleotide and predicted amino acid sequence of the truncated rat EGF-R. The numbers on the right correspond to the nucleotide sequence; the amino acid sequence is numbered throughout. Nonmatching amino acids in the human receptor are shown throughout the region of homology between the full-length and truncated receptors. Lowercase letters are used to indicate the region of the truncated receptor which is nonhomologous to the full-length receptor. The arrowhead indicates the point at which the homology between the full-length and truncated receptors ends. Nucleotide deletions in the human receptor sequence are indicated by black dots, and the star indicates an amino acid deletion. The polyadenylation signal and the 50-bp oligonucleotide used for Northern analysis and genomic cloning are underscored.

The DNA blots were probed with nick-translated cDNA insert from $\mathrm{pER} 1$ or $\mathrm{pER}$-t.

Immunoprecipitation. $\mathrm{CHO}$ cells were grown to confluence on $35-\mathrm{mm}$ plates. WB cells were grown to confluence on 100-mm plates. Cysteine- or methionine-free media alone or containing $10 \mu \mathrm{g}$ of tunicamycin per $\mathrm{ml}$ were added to the appropriate plates $1 \mathrm{~h}$ prior to the addition of label. Forty microcuries of $\left[{ }^{35} \mathrm{~S}\right]$ cysteine or $\left[{ }^{35} \mathrm{~S}\right]$ methionine per $\mathrm{ml}(\mathrm{Du}-$ Pont, NEN Research Products) was then added, and the cells were incubated for 2 to $3 \mathrm{~h}$. The media from all plates 


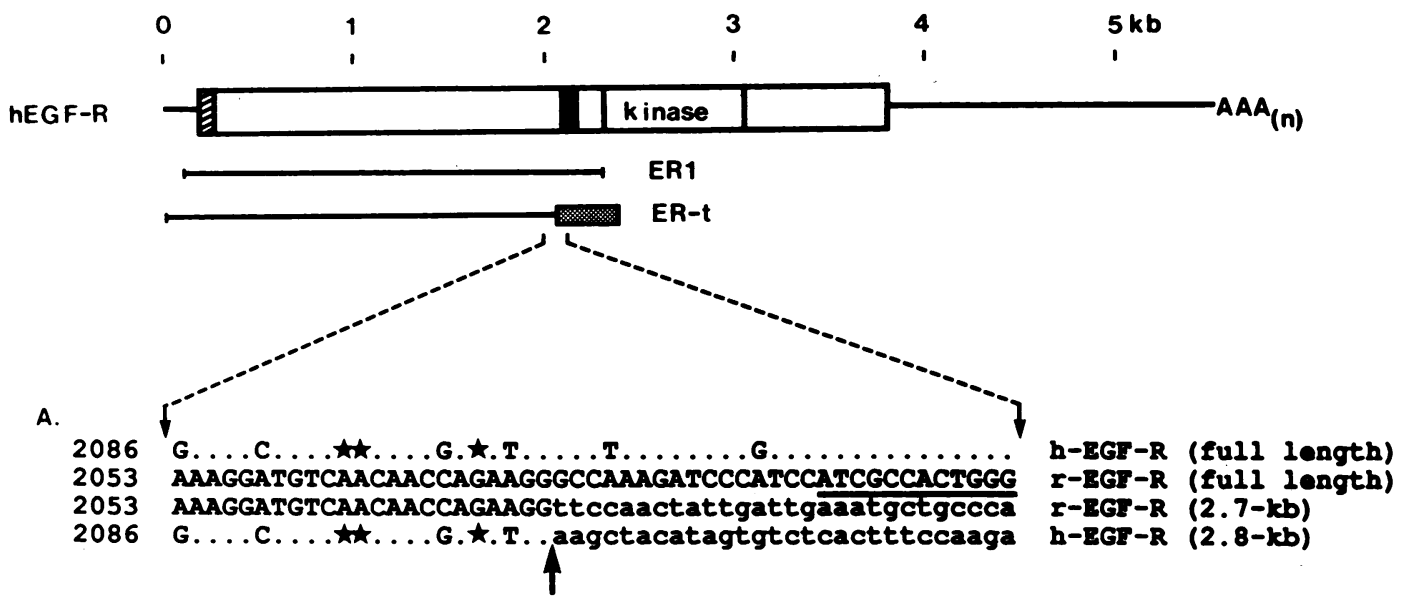

B.

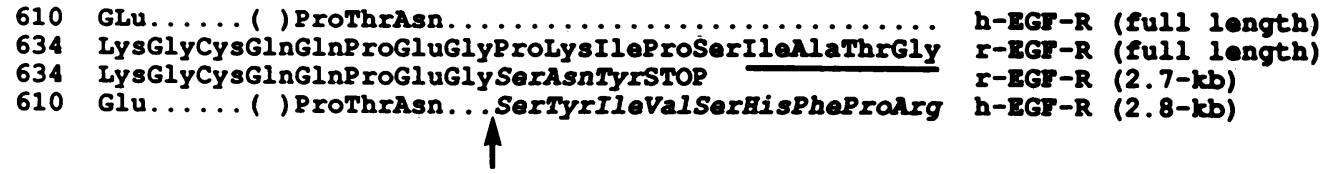

FIG. 3. Comparison of the human and rat full-length and truncated receptors at the junction of nonhomology. The arrow points to where the homology between the full-length and truncated receptors ends. The beginning of the transmembrane domain is underscored. (A) Nucleotide sequence comparison. Lowercase letters are used to indicate the divergent sequence of the truncated receptor. Dots represent identical nucleotides, and the nucleotide deletions in the human receptors are indicated by stars. (B) Amino acid sequence comparison. The italicized amino acids correspond to the divergent region of the truncated receptors. Dots represent identical amino acid residues, and the parentheses indicate the amino acid deletion. h-EGF-R, Human EGF-R; r-EGF-R, rat EGF-R.

were collected and, following detergent lysis of the cells, centrifugation, and preclearance of supernatants with normal rabbit serum and Pansorbin, the cells and media were immunoprecipitated as described previously (8). The polyclonal anti-rat EGF-R antiserum (Ab 1382) was raised in rabbits against purified rat liver EGF-R (8). Immunoprecipitates were boiled in electrophoresis sample buffer containing sodium dodecyl sulfate and were subjected to $7 \%$ polyacrylamide gel electrophoresis. Gels were treated for $1 \mathrm{~h}$ with $1 \mathrm{M}$ sodium salicylate and, after drying, were exposed to Kodak XAR film.

\section{RESULTS}

Cloning of EGF-R cDNAs. The isolation and characterization of a cDNA encoding a truncated form of the EGF-R in A431 epidermoid carcinoma cells have been previously described (43). This transcript was attributed to a rearrangement of the EGF-R gene in these cells. Here, we report the isolation of a similar cDNA encoding a secreted form of the EGF-R from normal rat liver. A rat liver cDNA library was screened with the human EGF-R clones $64-1$ and $64-3$ as hybridization probes. Positive clones were isolated and subcloned into the EcoRI site of pEMBL. Four of these were further analyzed by dideoxy sequencing. Comparison of the complete sequence of clone ER1, and a partial sequence of ER2 (see Fig. 1 for schematic representation), with that of the human EGF-R (43) revealed an overall similarity of 80 to $90 \%$. Relative to the human receptor sequence, the $2.1-\mathrm{kb}$ insert in plasmid pER1 extends from within the $5^{\prime}$ untranslated region to nucleotide $2317,92 \mathrm{bp}$ downstream from the end of the transmembrane coding region. The $0.7-\mathrm{kb}$ EcoRI insert in plasmid pER2 extends from nucleotide 2318 to nucleotide 3085 , spanning the tyrosine kinase domain of the EGF-R. Clones ER1 and ER2 are, therefore, contiguous and represent $>80 \%$ of the coding region of the EGF-R.

Nucleotide and amino acid sequence analysis. Examination of the nucleotide sequence of cDNA inserts ER-t and ER-ts revealed that they are independent clones derived from the same RNA. ER-ts, however, lacks 700 nucleotides from the $5^{\prime}$ terminus. The $2.3-\mathrm{kb}$ insert in plasmid pER-t contains 153 bp of 5' untranslated sequence and spans almost the entire extracellular domain of the receptor (Fig. 2 and 3). Relative to the membrane-spanning receptor cDNA ER1, the sequence homology of the two truncated cDNA clones declines abruptly at nucleotide 2105 , immediately prior to the transmembrane domain. This is followed by a stretch of 273 nucleotides which is identical in clones ER-t and ER-ts but which shares no homology with either rat or human EGF-R sequences. Furthermore, this stretch of sequence is distinct from the 3 '-terminal sequence of the $2.8-\mathrm{kb}$ transcript found in A431 cells (43). A computer search of sequences in GenBank and EMBL revealed no similarity to any other known sequences.

The predicted amino acid sequences of the membranespanning (ER1) and truncated (ER-t) EGF-R cDNAs reveal an open reading frame whose initiator methionine corresponds to the methionine at position -24 in the signal peptide of the human EGF-R (43). The sequence of clone ER-t is identical to that of clone ER1 through amino acid position 641 , at which point the sequence homology declines (Fig. 2 and 3). From residue 641, the open reading frame of the truncated receptor clone continues downstream for 


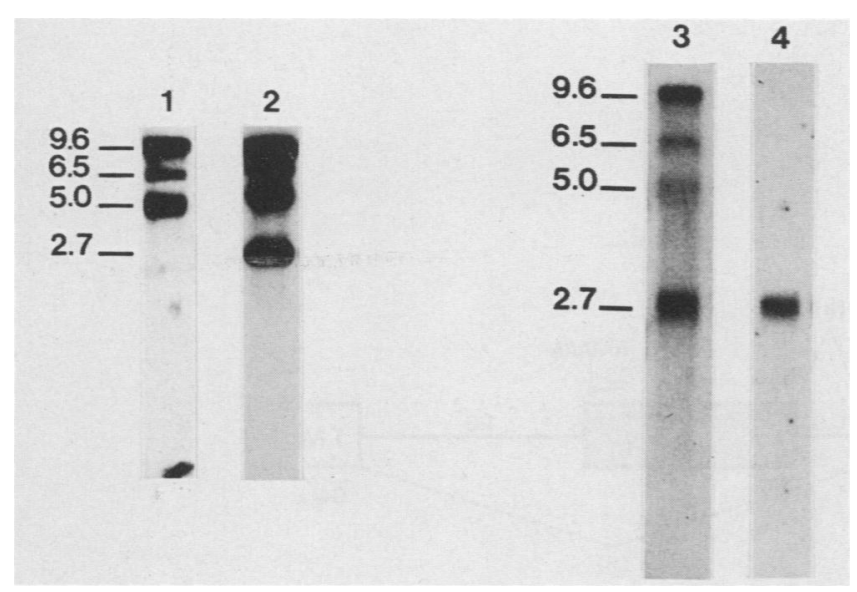

FIG. 4. Northern blot analysis of rat liver poly(A) ${ }^{+}$RNA. Poly $(\mathrm{A})^{+}$RNA was prepared, and samples $(5 \mu \mathrm{g})$ were electrophoresed through $1 \%$ agarose gels containing formaldehyde. RNA was transferred to nitrocellulose and was probed with nick-translated EcoRI inserts (Fig. 1) from plasmids pER2 (lane 1), pER-ts (lane 2), and pER1 (lane 3) or with an end-labeled 50-bp oligonucleotide (Fig. 2) specific for the 3'-end clones ER-t/ER-ts (lane 4). The numbers indicate (in kilobases) the size of the receptor transcripts.

another three amino acids and is then followed by a stop codon (TGA) and a 260-bp, 3' untranslated sequence, which includes a polyadenylation signal (AATAAA) 15 nucleotides upstream from the polyadenylation site.

A comparison of the amino acid sequence of the external domain of the rat and human EGF-R revealed an overall similarity of $94 \%$ when taking into account the fact that approximately one-half of the amino acid differences are conservative substitutions. Furthermore, alignment of these sequences through amino acid 636 (nucleotide 2061) revealed no deletions or insertions in either sequence (Fig. 2 and 3). Thus, all amino acid differences occurring throughout this region reflect base substitutions. However, immediately prior to the transmembrane domain of the human EGF-R, three nucleotide deletions occur within a 9-bp stretch of the sequence (Fig. 2 and 3A). This results in three amino acid changes and the loss of an amino acid from the otherwise highly conserved human receptor sequence (Fig. 3B). Interestingly, these three nucleotide deletions and the resulting amino acid changes occur immediately upstream from the point at which the sequence similarity between the truncated and full-length receptors ends.

Southern blot analysis. To determine whether the $3^{\prime}$ terminal sequence of cDNA clone ER-t is unique to the EGF-R gene, we carried out Southern blot analysis of rat genomic DNA with this cDNA as a hybridization probe. Three major bands $(5.1,3.3$, and $1.0 \mathrm{~kb})$ were consistently observed in $X b a I$-digested DNA with additional DNA fragments of 13.2, $11.0,4.2$, and $1.8 \mathrm{~kb}$ variably detected. Hybridization to EcoRI-digested genomic DNA consistently revealed a $3.3-\mathrm{kb}$ fragment. Additional bands (12.0, 8.8, 4.5, 2.4, and $2.2 \mathrm{~kb}$ ) were also occasionally detected. Identical restriction patterns were observed when either XbaI- or EcoRI-digested DNA was probed with the cDNA clone ER1 (data not shown). These findings confirm that the 3 '-terminal sequence of the truncated form of the EGF-R is present as a single copy sequence within the rat EGF-R gene.

Analysis of EGF-R mRNA expression. EGF-R synthesis in rat liver correlates with the detection of one major $(9.6 \mathrm{~kb})$ and two minor $(6.5$ and $5.0 \mathrm{~kb})$ species of RNA in total liver
RNA. However, Northern analysis of $\operatorname{poly}(A)^{+}$rat liver RNA with the human cDNA specific for the external domain of the receptor (64.1) as a hybridization probe revealed the presence of an additional $2.7-\mathrm{kb}$ receptor mRNA. This transcript was not observed when the cDNA specific for the tyrosine kinase domain (64.3) was used (data not shown). A 2.7-kb transcript is insufficient in size to code for the full-length EGF-R whose coding region alone spans approximately $3.6 \mathrm{~kb}$. To determine whether the truncated receptor cDNA described above is derived from this $2.7-\mathrm{kb}$ mRNA, we carried out Northern blot analyses of poly(A) ${ }^{+}$rat liver RNA with the rat cDNA clones as hybridization probes. Only the three larger EGF-R transcripts were detected with the insert from plasmid pER2, which, by comparison to the human EGF- $R$ sequence, is specific for the cytoplasmic tyrosine kinase domain (Fig. 4, lane 1). However, all four EGF-R transcripts were detected by clone ER-ts (Fig. 4, lane 2), which contains both homologous and 3' divergent sequences. The same result was obtained with clone ER1, which is specific for the external and transmembrane domain coding regions (Fig. 4, lane 3). These data show that the 2.7-kb mRNA in rat liver shares $5^{\prime}$ sequences, but not $3^{\prime}$ sequences, with the three larger receptor transcripts. This, in addition to the sequence data described above, suggests that clones ER-t and ER-ts, which lack the sequences coding for the transmembrane and cytoplasmic domains of the EGF-R are derived from the shorter, 2.7-kb receptor transcript. On the basis of the size discrepancy between the 2.3-kb ER-t cDNA and the 2.7-kb receptor transcript, it appears that the former is not a full-length clone. It is not clear, however, whether the ER-t cDNA is missing 5' or 3' sequences or whether the $2.7-\mathrm{kb}$ mRNA contains an unusually long poly(A) tract. To confirm that the ER-t and ER-ts cDNAs are derived from the $2.7-\mathrm{kb}$ mRNA, a 50-bp oligonucleotide, complementary to the 3' terminus of clone ER-t, was synthesized and used for Northern analysis. Only the 2.7-kb transcript was detected with the oligonucleotide probe (Fig. 4, lane 4). Furthermore, this oligonucleotide failed to hybridize to poly(A) ${ }^{+}$RNA from human A431 cells (data not shown), consistent with the sequence data that indicate that the rat and human truncated receptor transcripts possess distinct $3^{\prime}$ termini. The fact that the oligonucleotide probe, which is specific for the $3^{\prime}$ terminus of clone ER-t, hybridized only to the 2.7-kb EGF-R transcript provides additional evidence supporting the conclusion that the variant sequence is unique to the EGF-R gene.

Exon-intron analysis. A rearrangement of chromosome 7 has been postulated as the mechanism responsible for the generation of a truncated form of the EGF-R in human A431 cells (30). However, the data obtained thus far suggest that in normal rat liver cells, alternative splicing of the EGF-R gene is the mechanism by which this structural diversity is generated. To investigate this possibility, a rat genomic clone was isolated with both a truncated receptor-specific oligonucleotide and a 1.2-kb fragment of clone ER-t that contains both homologous and nonhomologous receptor sequences. Sequence analysis was carried out with the synthetic oligonucleotide primers shown in Fig. 5, and exon-intron junctions were identified by comparing genomic and cDNA sequences. The position of exons relative to each other was determined by analysis of the amplified products of the polymerase chain reaction carried out with these same primers (Fig. 5). The sequence data obtained confirm that the 3 '-terminal sequence of the truncated EGF-R is present as a specific exon in the EGF-R gene. These data also revealed that the last exon shared by the full-length and 
(a)

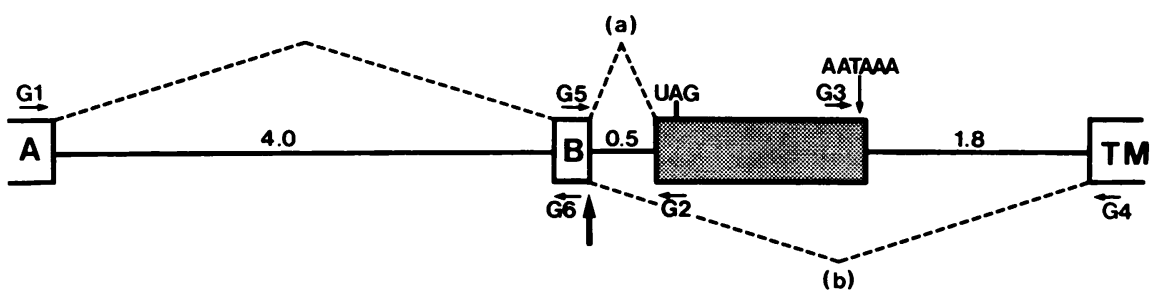

(b)

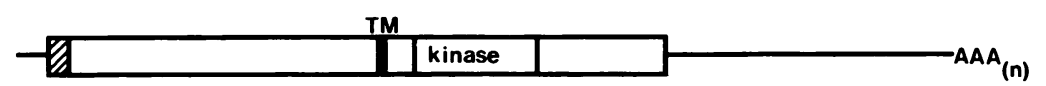

FIG. 5. Partial map of the exon-intron structure of the EGF-R gene and the splicing events which generate the truncated or full-length receptor transcripts. Exons A and B represent the last two exons shared by both the truncated and full-length receptors. The arrow indicates the junction of nonhomology between the full-length and truncated receptor transcripts. The stippled boxes represent the exon coding for the $3^{\prime}$ terminus of the $2.7-\mathrm{kb}$ receptor transcript. The stop codon and polyadenylation signal are shown. The exon labeled TM contains the transmembrane region of the full-length EGF-R. The synthetic oligonucleotides used to sequence the rat genomic clone are labeled G1 through G6. The hatched boxes at the 5' end of the coding region represent the signal peptide; the black box represents the transmembrane domain. The truncated receptor transcript resulting from one splicing event is shown at the top (a). The full-length receptor transcript resulting from the alternate splicing event is shown at the bottom (b). Numbers indicate (in kilobases) the approximate sizes of the introns.

truncated receptors consists of a 41-bp mini-exon that encodes the 14 amino acids at the C-terminal end of the extracellular domain (Fig. 5). It is interesting to note that the deletions observed when comparing the external domains of the human and rat receptors occur entirely within this mini-exon. Although this could be ascribed to a species difference, it also raises the possibility that there are EGF-R isoforms resulting from additional splicing events. For example, alternative splicing of a mini-exon generates isoforms of the human insulin receptor which differ in sequence at the C-terminal end of the insulin-binding $\alpha$-subunit $(36,37)$. As to the position of the rat EGF-R exons, the results obtained from the polymerase chain reaction analysis (Fig. 5) suggest that the ER-t-specific exon is preceded by the external domain mini-exon and lies upstream of that which codes for the transmembrane domain. As shown schematically in Fig. 5 , use of the ER-t-specific exon would result in the generation of a truncated EGF-R lacking transmembrane and intracellular receptor sequences.

Heterologous expression of the truncated receptor in $\mathrm{CHO}$ cells. To determine whether the $2.7-\mathrm{kb}$ transcript can indeed direct the synthesis of a truncated EGF-R protein, an expression vector containing the ER-t CDNA was introduced into CHO cells, which do not express EGF-Rs. The cells and conditioned growth media were subsequently analyzed for expression of this truncated protein by immunoprecipitation with antibody 1382, which recognizes the rat EGF-R. Immunoprecipitation of parental $\mathrm{CHO}$ cells labeled in the presence of tunicamycin, with normal rabbit serum or antibody 1382 (Fig. 6, lanes $A$ and $B$, respectively), failed to reveal a specific EGF-R-related protein. However, immunoprecipitation of stably transfected cells (CHO-8), labeled in the presence of tunicamycin, revealed a 65-kDa protein specifically recognized by antibody 1382 (lanes $C$ and $D$ ). A protein

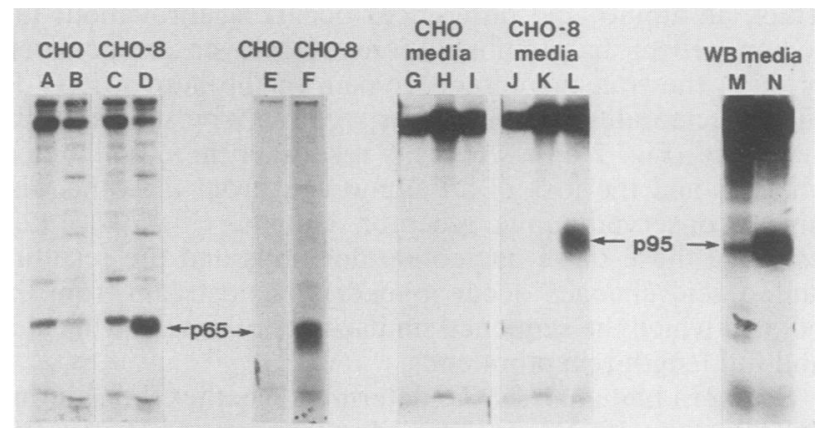

FIG. 6. Immunoprecipitation of the truncated EGF-R. CHO cells transfected with an expression vector containing cDNA clone ER-t were analyzed for expression of the truncated form of the EGF-R by immunoprecipitation of the cell lysates and conditioned media. The arrows indicate the migration of the truncated receptor protein. Control CHO (lanes A and B) or stably transfected CHO-8 (lanes $\mathrm{C}$ and D) cells were labeled with $\left[{ }^{35}\right.$ S]cysteine (lanes $\mathrm{A}$ through $D$ ) or $\left[{ }^{35} S\right]$ methionine (lanes $E$ and $F$ ) in the presence of tunicamycin. Cell lysates were immunoprecipitated as described previously (8) with normal rabbit serum (lanes A and C) or antiserum 1382 (lanes B and D). Media conditioned by control (lanes G through I) or transfected (lanes $J$ through $L$ ) cells, labeled with ${ }^{35}$ S ]cysteine in the presence (lanes $\mathrm{G}$ and $\mathrm{J}$ ) or absence (lanes $\mathrm{H}, \mathrm{I}$, $\mathrm{K}$, and $\mathrm{L}$ ) of tunicamycin, were immunoprecipitated by normal rabbit serum (lanes $H$ and $K$ ) or antiserum 1382 (lanes $G, I, J$, and L). Conditioned media from WB cells labeled in the absence of tunicamycin were immunoprecipitated with normal rabbit serum (lane M) or antiserum 1382 (lane $\mathrm{N}$ ). 


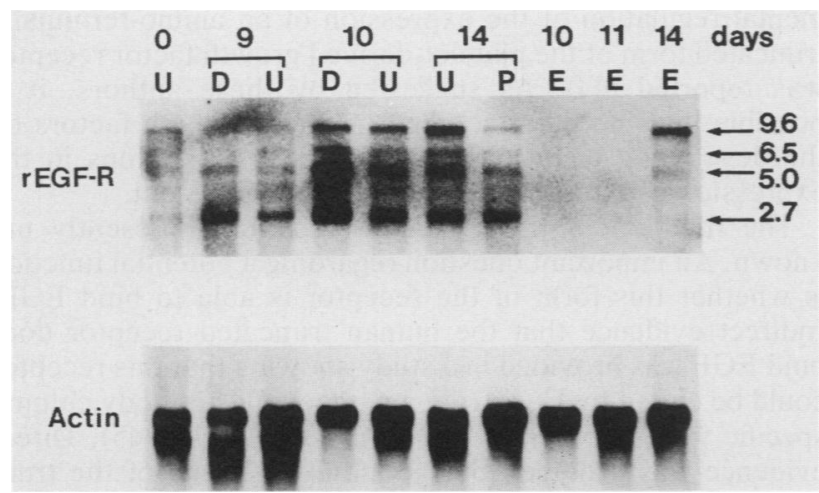

FIG. 7. Expression of the truncated rat EGF-R (rEGF-R) during rat development. Northern blot analysis of poly(A) ${ }^{+}$RNA from uterus (U), decidua (D), placenta (P), and embryo (E) at various times during gestation probed with cDNA clone ER-ts (Fig. 1) or actin. Arrows indicate the migration of the various EGF-R transcripts. Sizes are indicated in kilobases.

of similar size was also immunoprecipitated in these cells by normal rabbit serum (lane $C$ ). This protein appeared to be nonspecifically recognized, however, as evidenced by its presence in control cells immunoprecipitated with either normal rabbit serum or antibody 1382 (lanes A and B, respectively). Furthermore, this protein was not observed under all conditions. Immunoprecipitation with antibody 1382 of tunicamycin-treated $\mathrm{CHO}$ cells, labeled with ${ }^{35}$ S]methionine, failed to reveal any proteins in this size range (lane E). However, a $65-\mathrm{kDa}$ protein was detected by antibody 1382 in CHO-8 cells labeled with $\left[{ }^{35}\right.$ S $]$ methionine in the presence of tunicamycin (lane F).

Nucleotide and amino acid analysis of cDNA clone ER-t predicts it would encode a truncated form of the EGF-R which, because of the absence of its transmembrane and cytoplasmic domains, would be secreted. To test this hypothesis, we immunoprecipitated media conditioned by control and transfected cells. No EGF-R-related protein was detected in media conditioned by either tunicamycin-treated or untreated parental CHO cells (Fig. 6, lanes G and I, respectively). Furthermore, no receptor-related protein was detected in media conditioned by transfected cells treated with tunicamycin for $1 \mathrm{~h}$ prior to the addition of label (lane J). However, a 95-kDa protein was specifically detected by antibody 1382 in media conditioned by transfected cells labeled in the absence of tunicamycin treatment (lane L). These data suggest that the truncated form of the EGF-R is synthesized as a $65-\mathrm{kDa}$ precursor that is subsequently secreted as a $95-\mathrm{kDa}$ glycoprotein. To investigate whether this protein is synthesized by cells known to express the 2.7-kb EGF-R transcript, we immunoprecipitated media conditioned by WB cells, a nontransformed rat hepatic epithelial cell line. To compensate for the reduced level of expression of the truncated receptor in WB cells versus that in transfected $\mathrm{CHO}$ cells, five times the amount of media was used for the immunoprecipitation. A 95-kDa EGF-R-related protein is secreted by these cells (Fig. 6, lane N). Thus, this truncated form of the EGF-R does not reflect the transformed phenotype but is, in fact, secreted by normal proliferating epithelial cells known to express the $2.7-\mathrm{kb}$ transcript.

Previous studies have demonstrated that the truncated EGF-R secreted by human A431 cells binds EGF, albeit with a lower affinity than does the full-length receptor $(1,45)$. Preliminary cross-linking experiments show that a $95-\mathrm{kDa}$ protein, present in media conditioned by transfected but not parental CHO cells, is specifically labeled by $\left[{ }^{125} \mathrm{I}\right]-\mathrm{EGF}$ and recognized by antibody 1382 (data not shown).

Expression of EGF-R mRNAs in development. The expression of EGF and its receptor in development has been extensively studied (D. C. Lee and V. K. M. Han, in M. B. Sporn and A. B. Roberts, ed., Handbook of Experimental Pharmacology, in press). Therefore, it was of interest to examine the expression of the $2.7-\mathrm{kb}$ transcript during development. This transcript appears to be expressed at varying levels, during early gestation, in both the uterus and decidua, with the highest level of expression occurring in the decidua at day 10 (Fig. 7). In contrast, expression of receptor transcripts was not detected in fetal tissue until day 14 , at which time the full-length transcripts, but very low levels of the $2.7-\mathrm{kb}$ mRNA, were detected. With respect to adult tissues other than rat liver, EGF-R transcripts, including the 2.7-kb mRNA, were observed in lung tissue (data not shown).

\section{DISCUSSION}

The data presented here indicate that, in addition to the full-length EGF-R transcripts, normal rat liver cells express a shorter, 2.7-kb receptor mRNA. This transcript, generated by alternative splicing of the primary receptor mRNA, is homologous to the full-length EGF-R throughout the external hormone binding domain but lacks the transmembrane and tyrosine kinase domains and instead possesses a distinct 273-bp sequence at its extreme $3^{\prime}$ end. This divergent sequence introduces an in-frame stop codon immediately prior to the transmembrane region, generating a truncated EGF-R protein. As predicted, expression of cDNA clones of this transcript in CHO cells results in secretion of a $95-\mathrm{kDa}$ protein which is recognized specifically by antisera against the rat EGF-R. Our results also indicate that a similarly sized protein is secreted by WB cells, a line of normal rat hepatic epithelial cells, which also express the truncated $2.7-\mathrm{kb}$ EGF-R mRNA.

Altered expression of the EGF-R has been observed in several human tumors and tumor cell lines. For example, a receptor-related protein similar in size to this truncated receptor was recently observed in two human neuroblastoma cell lines (2). In addition, A431 human epidermoid carcinoma cells, which contain amplified and rearranged copies of the EGF-R gene, express an unusually high number of EGF-Rs and secrete a truncated form of the receptor $(9,30,43,45)$. This truncated species is encoded by a $2.8-\mathrm{kb}$ mRNA which is overexpressed in these cells $(30,43)$. It was proposed that this transcript is a hybrid RNA, resulting from an internal rearrangement of chromosome 7 , in which the $5^{\prime}$ portion encoding the outer domain of the EGF-R has been fused with unidentified DNA and subsequently amplified (30). It was, therefore, surprising to find that normal rat liver cells, in which the EGF-R gene is neither rearranged nor amplified, express a 2.7-kb mRNA which shares 5', but not 3', sequences with full-length EGF-R transcripts. A short transcript of similar size was also recently observed while studying EGF-R expression during rat liver regeneration (20). One possible explanation for the generation of this transcript is that this RNA is transcribed from a distinct, but related, gene. For example, a second platelet-derived growth factor receptor gene, which encodes a receptor exhibiting distinct platelet-derived growth factor binding characteristics, was recently described (29). Evidence has also been presented for multiple prolactin receptor mRNAs which 
appear to be encoded by at least two genes (6). That the truncated form of the EGF-R is not the product of a different gene is suggested by several lines of evidence. First, the full-length and truncated rat EGF-R clones are completely homologous throughout the external domain. Second, Southern blot analysis of rat liver genomic DNA with both the membrane spanning and truncated cDNAs confirms that the respective sequences are unique in the rat genome. Finally, sequence and polymerase chain reaction analyses of a rat genomic clone reveal that the point at which the full-length and truncated receptors diverge corresponds to an exon-intron junction. These results confirm, therefore, that the 3 '-terminal end of the 2.7 -kb transcript is present as a distinct exon in the EGF-R gene and that the truncated transcript is not the product of a different gene but is rather the result of alternative splicing of the primary EGF-R transcript.

The aforementioned results raise the possibility that the 2.8-kb A431 transcript is similarly generated by alternate splicing of unrearranged gene sequences. This hypothesis would not be inconsistent with previous data, which suggest that a chromosomal rearrangement gives rise to a truncated form of the EGF-R in A431 cells (30). Although the observed rearrangement was found to be associated only with a translocation marker chromosome (M4) present in these cells, it was not clear from these results whether the presence of this chromosome is responsible for the expression of the truncated receptor. Results obtained with mouse-human somatic cell hybrids do indicate, however, that this abnormal chromosome is clearly responsible for the hyperproduction of the EGF-R in A431 cells (38). It may also be responsible, therefore, for the overexpression of the truncated form of the EGF-R. Thus, it is possible that the observed rearrangement simply reflects the marker chromosome-associated alteration(s) involved in the amplification of the EGF-R gene as a whole rather than structural alterations within the gene itself. The hypothesis that the truncated EGF-R is a normal product of the EGF-R gene is further supported by the finding that the point at which the fulllength and truncated cDNAs diverge is identical in both humans and rats. Although the $3^{\prime}$ termini of the normal rat and human truncated receptor transcripts are apparently unrelated, this may simply reflect a lack of conservation among 3' sequences which are largely noncoding. However, the possibility that the truncated EGF-R transcript is generated by a fortuitous splicing event, resulting from a rearrangement that juxtaposed the human exon $B$ near a splice acceptor of another gene on chromosome 7 or led to the interruption of the normal receptor sequence by a novel DNA sequence, cannot be excluded.

The 2.7-kb EGF-R transcript is readily detected in rat liver mRNA, and its protein product is obviously produced and secreted by liver epithelial cells. In addition to rat liver, we have detected low-level expression of a 2.7-kb EGF-R mRNA in lung tissue and varying levels of expression in the uterus and decidua of pregnant rats. Although the truncated receptor transcript has only been observed in conjunction with the full-length receptor mRNAs, the expression of these transcripts is not always coregulated. For example, a significant elevation of the $2.7-\mathrm{kb}$ transcript was observed relative to the full-length EGF-R RNAs in day 10 decidua. Conversely, expression of the $2.7-\mathrm{kb}$ transcript was greatly reduced in day 14 embryonic tissue relative to the 9.6- and $5.0-\mathrm{kb}$ receptor transcripts. These data suggest that, consistent with a physiological role, the synthesis of the truncated receptor is developmentally regulated. Recently, develop- mental regulation of the expression of an amino-terminally truncated form of the platelet-derived growth factor receptor was reported (44). As suggested by these authors, it is possible that the putative effect of these growth factors on the developing embryo is modulated by alterations in the expression of their receptors during development.

The function of the truncated EGF-R is presently unknown. An important question regarding a potential function is whether this form of the receptor is able to bind EGF. Indirect evidence that the human truncated receptor does bind EGF was provided in a study showing that this receptor could be eluted by EGF from a monoclonal antibody column specific for the binding domain of the EGF-R (45). Direct evidence was provided by the affinity labeling of the truncated receptor with $\left.{ }^{125} \mathrm{I}\right]-\mathrm{EGF}$ (1). However, both of these studies revealed a 4 - to 20 -fold lower binding affinity than that observed for the full-length EGF-R. Preliminary results from affinity labeling studies in our laboratory indicate that the rat truncated receptor also binds EGF. Thus, one possibility consistent with the secretion of this form of the receptor is that it could act as a serum binding protein. Such a function has been ascribed to a secreted form of the growth hormone receptor that is also generated by alternative splicing $(25,39)$. Alternatively, it could modulate the response of target cells to EGF and transforming growth factor alpha by competing for these ligands. This is an interesting possibility in light of a recent report of an astrocyte mitogen inhibitory activity detected in rat brain and liver extracts (33). This activity could be abrogated by anti-EGF-R antibodies, suggesting that the inhibitor is immunologically related to the EGF-R. These data are consistent with the hypothesis that a secreted form of the EGF-R could modulate normal EGF-R activity by competition for ligand. A recent study of the A431 truncated receptor suggests that it not only binds to EGF but also interacts with full-length receptors to inhibit activation of the tyrosine kinase. However, this effect was not attributable to competition for ligand (1). Thus, while the truncated form of the EGF-R may alter the response of cells to ligand, it may do so through a mechanism other than simple competition. Recently, an isoform of the thyroid hormone receptor generated by alternative splicing was described (19). This form of the receptor is unable to bind thyroid hormone or activate thyroid hormone-responsive genes. However, coexpression of the active and inactive forms of the thyroid hormone receptor led to inhibition of the thyroid hormone-inductive effect of the former (21). Formation of nonfunctional heterodimers of the active and inactive thyroid hormone receptors was suggested as one possible mechanism responsible for this inhibitory effect. It was further proposed that inhibition of hormone action by a splicing variant of a receptor is a novel mechanism for the modulation of hormone responsiveness. The truncated form of the EGF-R could represent another example of such a regulatory mechanism.

\section{ACKNOWLEDGMENTS}

We thank Alice Berry, Debra Hunter, and Lisa Winchell for experimental assistance. We are indebted to Jane Radford for skillful preparation of this manuscript.

This work was supported by Public Health Service grants DK30002 (H.S.E.) and CA-43793 (D.C.L.) from the National Institutes of Health.

\section{LITERATURE CITED}

1. Basu, A., M. Raghunath, S. Bishayee, and M. Das. 1989. Inhibition of tyrosine kinase activity of the epidermal growth 
factor (EGF) receptor by a truncated receptor form that binds to EGF: role for interreceptor interaction in kinase regulation. Mol. Cell. Biol. 9:671-677.

2. Brown, A., and G. Carpenter. 1988. Presence of altered epidermal growth factor receptor expression in human neuroblastoma cells. FASEB J. 2:A1774.

3. Carpenter, G. 1987. Receptors for EGF and other polypeptide mitogens. Annu. Rev. Biochem. 56:881-914.

4. Carpenter, G., and S. Cohen. 1979. Epidermal growth factor. Annu. Rev. Biochem. 48:193-216.

5. Chirgwin, J. M., A. E. Przybyla, R. J. McDonald, and W. J. Rutter. 1979. Isolation of biologically active ribonucleic acid from sources enriched in ribonuclease. Biochemistry 18:5294-5299.

6. Davis, J. A., and D. I. H. Linzer. 1989. Expression of multiple forms of the prolactin receptor in mouse liver. Mol. Endocrinol. 3:674-680.

7. Downward, J., Y. Yarden, E. Mayes, G. Scrace, N. Totty, P. Stockwell, A. Ullrich, J. Schlessinger, and M. D. Waterfield. 1984. Close similarity of epidermal growth factor receptor and v-erb-B oncogene protein sequences. Nature (London) 307: 521-527.

8. Earp, H. S., K. S. Austin, J. Blaisdell, R. A. Rubin, K. G. Nelson, L. W. Lee, and J. W. Grisham. 1986. Epidermal growth factor (EGF) stimulates EGF receptor synthesis. J. Biol. Chem. 261:4777-4780

9. Fabricant, R. N., J. E. DeLarco, and G. J. Todaro. 1977. Nerve growth factor receptor on human melanoma cells in culture. Proc. Natl. Acad. Sci. USA 74:565-569.

10. Filmus, J., M. N. Pollak, J. G. Cairncross, and R. N. Buick. 1985. Amplified, overexpressed and rearranged epidermal growth factor receptor gene in a human astrocytoma cell line. Biochem. Biophys. Res. Commun. 131:207-215.

11. Fung, Y. T., W. G. Lewis, L. B. Crittenden, and H. Kung. 1983. Activation of the cellular oncogene c-erbB by LTR insertion: molecular basis for induction of erythroblastosis by avian leukosis virus. Cell 33:357-368.

12. Glisin, V., R. Crkvenjakov, and C. Byus. 1974. Ribonucleic acid isolated by cesium chloride centrifugation. Biochemistry 13: 2633-2637.

13. Graf, T., and H. Beug. 1983. Role of the v-erbA and v-erbB oncogenes of avian erythroblastosis virus in erythroid cell transformation. Cell 34:7-9.

14. Graham, F. L., and A. J. van der Eb. 1973. A new technique for the assay of infectivity of human adenovirus 5 DNA. Virology 52:456-467.

15. Gullick, W. J., J. J. Marsden, N. Whittle, B. Ward, L. Bobrow, and M. Waterfield. 1986. Expression of epidermal growth factor receptors on human cervical, ovarian and vulval carcinomas. Cancer Res. 46:285-292.

16. Hayman, M. J., G. M. Ramsey, K. Savin, G. Kitchener, T. Graf, and H. Beug. 1983. Identification and characterization of the avian erythroblastosis virus erbB gene product as a membrane glycoprotein. Cell 32:579-588.

17. Hendler, F. J., and B. W. Ozanne. 1984. Human squamous cell lung cancers express increased epidermal growth factor receptors. J. Clin. Invest. 74:647-651.

18. Hunts, J., M. Ueda, S. Ozawa, O. Abe, I. Pastan, and N. Shimizu. 1985. Hyperproduction and gene amplification of the epidermal growth factor receptor in squamous cell carcinomas. Jpn. J. Cancer Res. 76:663-666.

19. Izumo, S., and V. Mahdavi. 1988. Thyroid hormone receptor $\alpha$ isoforms generated by alternative splicing differentially activate myosin HC gene transcription. Nature (London) 334:539-542.

20. Johnson, A. C., S. H. Garfield, G. T. Merlino, and I. Pastan. 1988. Expression of epidermal growth factor receptor protooncogene mRNA in regenerating rat liver. Biochem. Biophys. Res. Commun. 150:412-418.

21. Koenig, R. J., M. A. Lazar, R. A. Hodin, G. A. Brent, P. R. Larsen, W. W. Chin, and D. D. Moores. 1989. Inhibition of thyroid hormone action by a non-hormone binding c-erbA protein generated by alternative mRNA splicing. Nature (London) 337:659-661.

22. Kris, R. M., I. Lax, W. Gullick, M. D. Waterfield, A. Ullrich, M.
Fridkin, and J. Schlessinger. 1985. Antibodies against a synthetic peptide as a probe for the kinase activity of the avian EGF receptor and v-erbB protein. Cell 40:619-625.

23. Lax, I., R. Kris, I. Sasson, A. Ullrich, M. J. Hayman, H. Beug, and J. Schlessinger. 1985. Activation of c-erbB in avian leukosis virus-induced erythroblastosis leads to the expression of a truncated EGF receptor kinase. EMBO J. 4:3179-3182.

24. Lee, D. C., T. M. Rose, N. R. Webb, and G. J. Todaro. 1985. Cloning and sequence analysis of a cDNA for rat transforming growth factor $\alpha$. Nature (London) 313:489-491.

25. Leung, D. W., S. A. Spencer, G. Cachianes, R. G. Hammonds, C. Collins, W. J. Henzel, R. Barnard, M. J. Waters, and W. I. Wood. 1987. Growth hormone receptor and serum binding protein: purification, cloning and expression. Nature (London) 330:537-543.

26. Libermann, T. A., H. R. Nusbaum, N. Razon, R. Kris, I. Lax, H. Soreq, N. Whittle, M. D. Waterfield, A. Ullrich, and J. Schlessinger. 1985. Amplification, enhanced expression and possible rearrangement of EGF receptor gene in primary human brain tumors of glial origin. Nature (London) 313:144-147.

27. Lin, C. R., W. S. Chen, W. Kruiger, L. S. Stolarsky, W. Weber, R. Evans, I. Verma, G. N. Gill, and M. G. Rosenfeld. 1984 Expression cloning of human EGF receptor complementary DNA: gene amplification and three related messenger RNA products in A431 cells. Science 224:843-848.

28. Maihle, N. J., and H.-J. Kung. 1988. C-erbB and the epidermal growth factor receptor: a molecule with dual identity. Biochim. Biophys. Acta 948:287-304.

29. Matsui, T., M. Heidaran, T. Miki, N. Popescu, W. La Rochelle, M. Kraus, J. Pierce, and S. Aaronson. 1989. Isolation of a novel receptor CDNA establishes the existence of two PDGF receptor genes. Science 243:800-804.

30. Merlino, G. T., S. Ishii, J. Whang-Peng, T. Knutsen, Y.-H. Xu, A. J. L. Clark, R. H. Stratton, R. K. Wilson, D. P. Ma, B. A. Roe, J. H. Hunts, N. Shimizu, and I. Pastan. 1985. Structure and localization of genes encoding aberrant and normal epidermal growth factor receptor RNAs from A431 human carcinoma cells. Mol. Cell. Biol. 5:1722-1734.

31. Merlino, G. T., Y. Xu, S. Ishii, A. J. L. Clark, K. Semba, K. Toyoshima, T. Yamamoto, and I. Pastan. 1984. Amplification and enhanced expression of the epidermal growth factor receptor gene in A431 human carcinoma cells. Science 224:417-419.

32. Miles, B. D., and H. L. Robinson. 1985. High-frequency transduction of c-erbB in avian leukosis virus-induced erythroblastosis. J. Virol. 54:295-303.

33. Nieto-Sampedro, M. 1988. Astrocyte mitogen inhibitor related to epidermal growth factor receptor. Science 240:1784-1786.

34. Ozanne, B., C. S. Richards, F. Hendler, D. Burns, and B. Gusterson. 1986. Over-expression of the EGF receptor is a hallmark of squamous cell carcinomas. J. Pathol. 149:9-14.

35. Sanger, F., S. Nicklen, and A. R. Coulson. 1977. DNA sequencing with chain-terminating inhibitors. Proc. Natl. Acad. Sci. USA 74:5463-5467.

36. Seino, S., and G. I. Bell. 1989. Alternative splicing of human insulin receptor messenger RNA. Biochem. Biophys. Res. Commun. 159:312-316.

37. Seino, S., M. Seino, S. Nishi, and G. I. Bell. 1989. Structure of the human insulin receptor gene and characterization of its promoter. Proc. Natl. Acad. Sci. USA 86:114-118.

38. Shimizu, N., I. Kondo, S. Gamou, M. A. Behzadian, and Y. Shimizu. 1984. Genetic analysis of hyperproduction of epidermal growth factor receptors in human epidermoid carcinoma A431 cells. Somatic Cell Genet. 1:45-53.

39. Smith, W. C., D. I. H. Linzer, and F. Talamantes. 1988. Detection of two growth hormone receptor mRNAs and primary translation products in the mouse. Proc. Natl. Acad. Sci. USA 85:9576-9579.

40. Southern, E. M. 1975. Detection of specific sequences among DNA fragments separated by gel electrophoresis. J. Mol. Biol. 98:503-517.

41. Steffens, D. L., and R. W. Gross. 1989. Sequencing of cloned DNA using bacteriophage $\lambda$ gt11 templates. Biotechniques 7: 674-679. 
42. Thomas, P. S. 1980. Hybridization of denatured RNA and small DNA fragments transferred to nitrocellulose. Proc. Natl. Acad. Sci. USA 77:5201-5205.

43. Ullrich, A., L. Coussens, J. S. Hayflick, T. J. Dull, A. Gray, A. W. Tam, J. Lee, Y. Yarden, T. A. Libermann, J. Schlessinger, J. Downward, E. L. V. Mayes, N. Whittle, M. D. Waterfield, and P. H. Seeburg. 1984. Human epidermal growth factor receptor cDNA sequence and aberrant expression of the amplified gene in A431 epidermoid carcinoma cells. Nature (London) 309:418-425.

44. Vu, T. H., G. R. Martin, P. Lee, D. Mark, A. Wang, and L. T. Williams. 1989. Developmentally regulated use of alternative promoters creates a novel platelet-derived growth factor receptor transcript in mouse teratocarcinoma and embryonic stem cells. Mol. Cell. Biol. 9:4563-4567.

45. Weber, W., G. N. Gill, and J. Spiess. 1984. Production of an epidermal growth factor receptor-related protein. Science 224: 294-297.
46. Wigler, M., S. Silverstein, L.-S. Lee, A. Pellicer, Y.-C. Cheng, and R. Axel. 1977. Transfer of purified herpes virus thymidine kinase gene to cultured mouse cells. Cell 11:223-232.

47. Xu, Y., S. Ishii, A. J. L. Clark, M. Sullivan, R. K. Wilson, D. P. Ma, B. A. Roe, G. T. Merlino, and I. Pastan. 1984. Human epidermal growth factor receptor cDNA is homologous to a variety of RNAs overproduced in A431 carcinoma cells. Nature (London) 309:806-810.

48. Yamamoto, T., N. Kamata, H. Kawano, S. Shimizu, T. Kuroki, K. Toyoshima, K. Rikimaru, N. Nomura, R. Ishizaki, I. Pastan, S. Gamou, and N. Shimizu. 1986. High incidence of amplification of the epidermal growth factor receptor gene in human squamous carcinoma cell lines. Cancer Res. 46:414-416.

49. Yamamoto, T., T. Nishida, N. Miyajima, S. Kawai, T. Ooi, and K. Toyoshima. 1983. The erbB gene of avian erythroblastosis virus is a member of the src gene family. Cell 35:71-78.

50. Yarden, Y., and A. Ullrich. 1988. Growth factor receptor tyrosine kinases. Annu. Rev. Biochem. 57:443-478. 